\title{
Therapeutic Hypothermia Still Effective in Prevention of Anoxic Encephalop- athy following Extended Period of Pulselessness during Cardiac Arrest
}

\author{
Rivera $\mathrm{NT}^{*}$, Bray $\mathrm{N}^{1}$, Osman $\mathrm{A}^{2}$, Kumar $\mathrm{S}^{3}$ and Vicuña $\mathrm{R}^{4}$ \\ ${ }^{1}$ Department of Internal Medicine, Broward Health Medical Center, Fort Lauderdale, FL, USA \\ ${ }^{2}$ Department of Cardiology, Electrophysiology, Broward Health Medical Center, Fort Lauderdale, FL, USA \\ ${ }^{3}$ Department of Pulmonary and Critical Care Medicine, Broward Health Medical Center, Fort Lauderdale, FL, USA \\ ${ }^{4}$ Department of Cardiology, Interventional, Broward Health Medical Center, Fort Lauderdale, FL, USA
}

${ }^{*}$ Corresponding author: Rivera NT, DO/PhD, Department of Internal Medicine, Broward Health Medical Center, 1600 South Andrews Avenue, Fort Lauderdale, FL 33316, E-mail: N1rivera@browardhealth.org

Citation: Rivera NT, Bray N, Osman A, Kumar S, Vicuña R (2014) Therapeutic Hypothermia Still Effective in Prevention of Anoxic Encephalopathy following Extended Period of Pulselessness during Cardiac Arrest. J Clin Exp Res Cardiol 1(1): 101. doi: 10.15744/2394-6504.1.101

Received Date: January 30, 2014 Accepted Date: February 05, 2014 Published Date: February 10, 2014

\begin{abstract}
There are approximately 300,000 out-of-hospital cardiac arrests per year with less than $10 \%$ of those surviving. More than half of survivors suffer permanent neurologic deficits. Therapeutic hypothermia has proven effective at thwarting neurologic damage occurring in the 16-hour window following return of spontaneous circulation (ROSC). Despite recommendations by the American Heart Association (AHA), many cardiologists have been slow to implement therapeutic hypothermia. While many trials have discussed the relevance of initial rhythm and delay of cooling, there has been limited discussion of the efficacy of therapeutic hypothermia in the presence of extended pulselessness.

A 40-year-old male with past medical history of an ST-segment elevation myocardial infarction 8 years prior, presented with an out-ofhospital cardiac arrest. Cardiopulmonary resuscitation was immediately initiated. The patient remained in ventricular fibrillation for 50 minutes with only one episode of a momentary pulse prior to persistent ROSC.

Cardiac catheterization demonstrated $100 \%$ occlusion of the right coronary artery, which was unable to be revascularized. Numerous collateral branches were present and the ejection fraction was significantly reduced at $10 \%$. An intra-aortic balloon pump was inserted; he was initiated on vasopressors and continued on therapeutic hypothermia. Following passive re-warming, he was found to be neurologically intact and continued to be without deficit both throughout his 7-week hospitalization and outpatient follow-up for 8

In this case, we describe the full neurologic recovery following cardiac arrest with essentially 50 minutes without a sustained pulse through the employment of therapeutic hypothermia. This report demonstrates the superior utility of therapeutic hypothermia even in the setting of extended-period pulselessness and its positive impact on both morbidity and mortality. The course of this patient's treatment further support the AHA recommendations and will be a useful adjunct to the current literature for determining treatment and prognosis in similar cases.
\end{abstract}

Keywords: Cardiac arrest; Therapeutic hypothermia; Sudden cardiac death; Acute coronary syndrome; Cardiopulmonary resuscitation; Myocardial infarction

\section{Introduction}

According to the American Heart Association (AHA), in 2013 the incidence of out-of-hospital cardiac arrest was 359,400, of which the overall survival rate was $9.5 \%$ [1], with greater than $50 \%$ of those survivors suffering the sequelae permanent neurologic deficits [2]. Neurologic damage commonly occurs through a combination of absence of cerebral oxygenation during pulselessness and reperfusion injury following return of spontaneous circulation (ROSC) [3].

In 2002, the Advanced Life Support (ALS) Task Force of the International Liaison Committee on Resuscitation (ILCOR) introduced the new recommendations regarding the use of therapeutic hypothermia following ROSC in the event of cardiac arrest [4] Based on the evidence from two landmark prospective randomized clinical trials, therapeutic hypothermia has proven effective in preventing crippling neurologic injury in the 16-hour window following ROSC [5,6], with $92 \%$ demonstrating a positive neurologic outcome [7].

Many clinical trials have examined the effectiveness of therapeutic hypothermia on different parameters such as the initial rhythm during cardiac arrest, time delay after ROSC in initiating cooling, and length of time in hypothermic state [4,7]. However, discussion 
regarding pulselessness and its effect on neurologic recovery through the use of therapeutic hypothermia is limited. Despite the overwhelming evidence supporting the superior effect of therapeutic hypothermia on mortality, many cardiologists are slow to implement its practice [7].

In this case report, we describe the complete neurologic recovery following cardiac arrest with essentially 50 minutes without a sustained pulse through the utilization of therapeutic hypothermia.

\section{Case Report}

A 40-year-old male with past medical history of an ST-segment elevation myocardial infarction (STEMI) at the age of 32-yearsold with subsequent placement of two drug eluting stents (DES) to the right coronary artery (RCA) had a witnessed syncopal event in his home. Bystander cardiopulmonary resuscitation (CPR) was immediately initiated by family, alongside notification of emergency medical services (EMS). Paramedics found an initial rhythm of ventricular fibrillation (VF) and advanced cardiovascular life support (ACLS) was executed as he was transported to the emergency department. The patient remained in VF for 30 minutes prior to a momentary ROSC. CPR was continued as the patient's rhythm reverted to VF for another 20 minutes prior to persistent ROSC, resulting in a total of 50 minutes of CPR without a sustained pulse.

Immediately following persistent ROSC, the patient was initiated on therapeutic hypothermia and sent for cardiac catheterization. Within the left coronary system no significant coronary artery disease was found (Figure 1a). The RCA was 100\% occluded proximally and unyielding to multiple attempts at passage of a guide wire for purposes of revascularization (Figure 1b). There were numerous left to right collaterals of the left coronary system within the posterior descending artery (PDA) and posterior lateral branch (Figures 1c, 1d \& 1e). The ejection fraction was significantly reduced at 10\% (Figure 2).
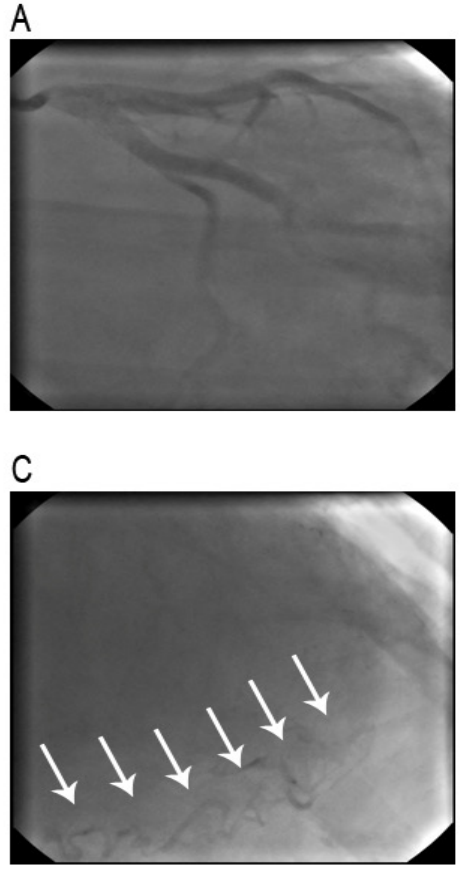

E

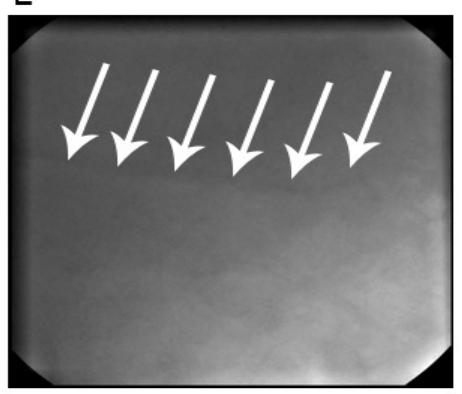

B

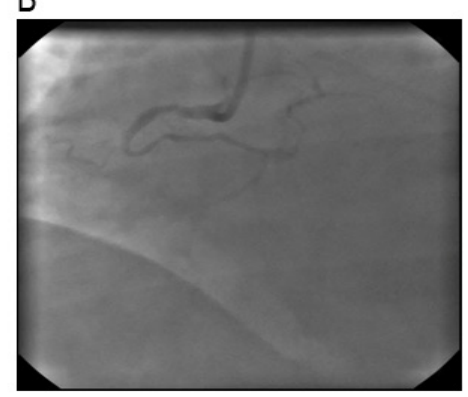

D

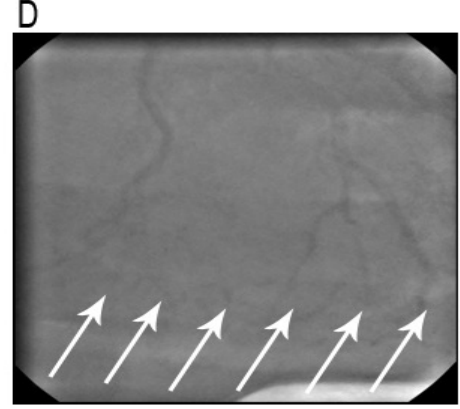

F

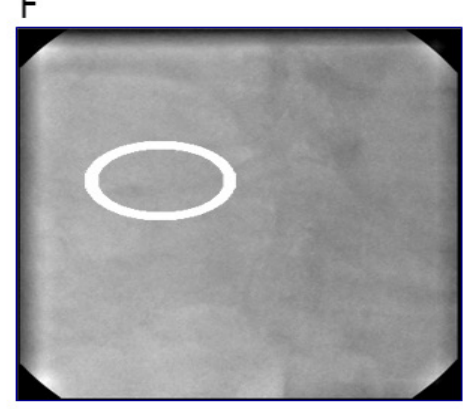

Figure 1: Cardiac catheterization of the left coronary system (A) and right coronary system (B) demonstrating complete occlusion of the proximal portion of the right coronary artery (RCA). Cardiac catheterization establishing the presence of left to right collateral branches (C and D, indicated by arrows) with filling of the distal RCA (E, indicated by arrows). Previously inserted drug eluting stent can be seen in the distal RCA (F, indicated by oval). 
A
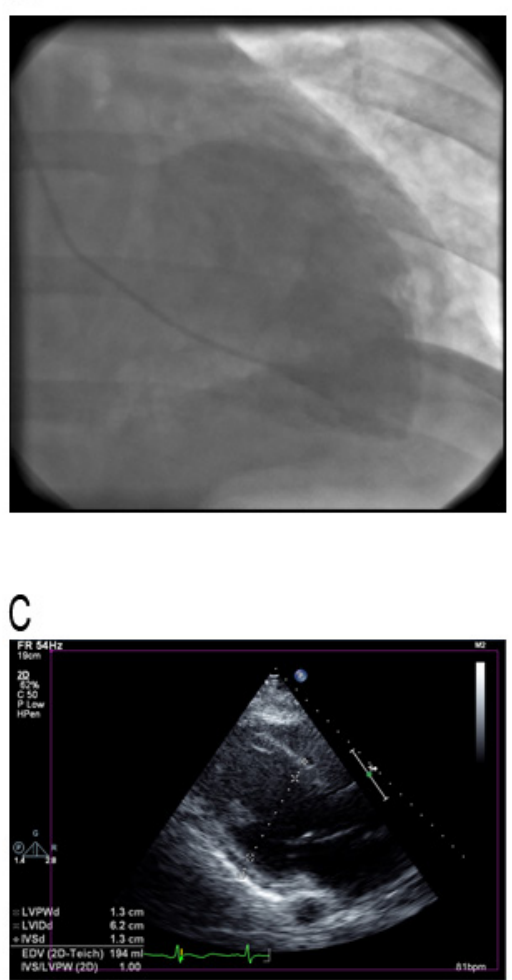

B

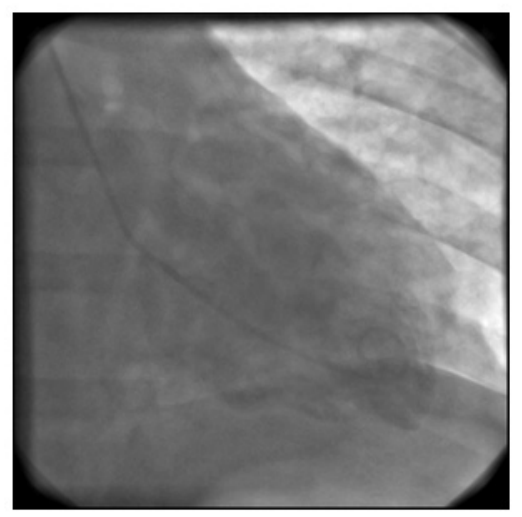

D

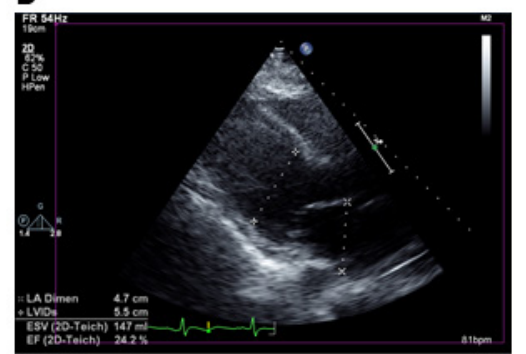

Figure 2: Cardiac catheterization $(\mathrm{A}$ and $\mathrm{B})$ and transthoracic echocardiogram $(\mathrm{C}$ and $\mathrm{D})$ demonstrating severe global hypokinesis of the left ventricle with a significantly reduced ejection fraction of $10 \%$ by comparison of systole (A and C) and diastole (B and $\mathrm{D})$.

Preliminary steps in temperature reduction included the use of external cooling packs and a cooling blanket in the process of being transferred for cardiac catheterization. Following angiographic evaluation of the coronary arteries, an Icy ${ }^{\mathrm{TM}} \mathrm{catheter} \mathrm{was} \mathrm{inserted}^{\circ}$ and connected to a CoolGard ${ }^{\mathrm{TM}}$ system. The patient was cooled with intravenous fluids from $37^{\circ} \mathrm{C}$ to $32^{\circ} \mathrm{C}$ over a total period of 6-hours. An intra-aortic balloon pump (IABP) was also inserted and he was initiated on vasopressors. The patient was continued on therapeutic hypothermia and maintained at a core bladder temperature of $32{ }^{\circ} \mathrm{C}$ for 24 -hours. Passive re-warming was achieved over a period of 6-hours at approximately $0.5^{\circ} \mathrm{C}$ per hour. The CoolGard ${ }^{\mathrm{TM}}$ system remained connected to maintain the patient in normothermia for the subsequent 48-hours, however it was not required during this case. Follow-up evaluation confirmed the patient to be completely neurologically intact and continued to be without deficit both throughout his 7-week hospitalization and outpatient follow-up for 8 months.

Throughout his hospital course, the patient remained in critical condition with multiple complications. He became volumeoverloaded secondary to acute kidney failure with anuria and was treated with continuous veno-venous hemodialysis.. He acquired ventilator-associated pneumonia (VAP) with both Pseudomonas aeruginosa and methicillin-resistant Staphylococcus aureus and was treated with vancomycin and piperacillin-tazobactam. He remained on vasopressors for a week and had intermittent episodes of atrial fibrillation with rapid ventricular response. He was initially intubated and then subsequently received a tracheostomy for a total of 6 weeks. His kidneys recovered fully and the tracheostomy was successfully decannulated following resolution of VAP. Due to his continued arrhythmias in the presence of treatment with amiodarone coupled with his established severe ischemia cardiomyopathy, a dual chamber implantable cardioverter-defibrillator was inserted. The patient underwent physical therapy with cardiac rehabilitation and had a full neurologic recovery without sequelae.

\section{Discussion}

In 2013, there were more than half of a million cardiac arrests in the United States, 209,000 and 359,400, in- and out-of-hospital respectively [1]. Survival rates are quite dismal at $23.9 \%$ and $9.5 \%$, in- and out-of-hospital respectively [1]. Permanent neurologic damage following survival has a high incidence at greater than 50\% [2]. Neurologic damage occurs through multiple mechanisms. During cardiac arrest, there is a lack of oxygen, which results in a direct loss of adenosine tri-phosphate (ATP) causing elevated intracellular calcium thereby increasing free radicals. This in turn disrupts the energy-producing capability of the cell resulting in direct cell death [8-10]. Reperfusion injury following ROSC involves an inflammatory response resulting in the release of

cytokines and creation of free radicals. Free radicals are enzymatically combined with the now-available oxygen forming reactive oxygen species, which directly damages cell components yielding cell death [11,12]. Even with survival following cardiac arrest, returning to a pre-arrest functional state is improbable. 
In 2002, two landmark prospective randomized clinical trials published their results effectively changing both morbidity and mortality following cardiac arrest $[5,6]$. Based on these trials, the ALS Task Force of the ILCOR issued new recommendations regarding care and management following ROSC in the event of cardiac arrest [4]. In the European study, in the hypothermia group, the median time until ROSC was 21 minutes and the interquartile range was $17-28$ minutes [6]. Statistical analysis concluded for every additional 90 seconds until ROSC, there was a decrease in the likelihood of a good outcome (survival to hospital discharge and positive neurologic outcome based on cerebral performance category) by 14\% [6]. Of note, in this study, bystander CPR did not alter the outcome [6]. In the Australian study, the mean time until ROSC was 26.5 minutes with a standard deviation of 12.9 minutes [5]. The majority of patients who experienced neurologic deficits died within 6 months after cardiac arrest [5]. Both studies demonstrated better neurologic outcome in the hypothermia group as compared to the normothermia group [5,6].

Since the new recommendations for therapeutic hypothermia have been released in 2002, multiple trials have been conducted to further examine the optimal parameters to put forth a protocol for enhanced neurologic outcome. In one such trial, the median time from arrest to ROSC was 22 minutes [7]. This study demonstrated a strong association between reduced time until ROSC and better chance for survival [7]. Further, only 36\% of patients survived when the time until ROSC was greater than 30 minutes [7]. Of note, they also concluded that increased mortality was seen in cases of advanced age, asystole/pulseless electrical activity as initial rhythm, and cardiogenic shock [7]. However, only advanced age, and not initial rhythm or associated cardiogenic shock, was associated with permanent neurologic damage [7]. Similar to previous studies, delay of therapeutic hypothermia induction significantly impacted survival [7,13-16]. The risk of death escalated by $20 \%$ for every hour delay in initiation of cooling [7]. Still, overall, multiple trials have found a reduction in mortality by $35 \%$ through employment of therapeutic hypothermia [17].

Despite the overwhelming evidence in favor of therapeutic hypothermia following cardiac arrest as a means of "minimizing morbidity and mortality, many cardiologists have been hesitant to implement it. Concern may be present with the practical utility of therapeutic hypothermia following extended downtime prior to ROSC. While many large-scale studies have indicated decreased benefit in response after greater than 30 minutes delay of ROSC, the current case report demonstrates that in fact both survival and full neurologic recovery can be achieved. Further, many studies have indicated that it is the postponement in initiating cooling that results in decreased efficacy of therapeutic hypothermia. In the present case, there was negligible delay time between ROSC and initiation of hypothermia. Another favorable factor for full neurologic recovery in this patient can be attributed to his younger age.

\section{Conclusions}

Multiple trials have been conducted to evaluate the impact of therapeutic hypothermia on cardiac arrest morbidity and mortality. These studies have found that shorter periods of pulselessness contributed to better outcomes. In the current case report, we demonstrate the full neurologic recovery following 50 minutes without a sustained pulse during cardiac arrest through the utility of therapeutic hypothermia. This case demonstrates that even with extended pulselessness, therapeutic hypothermia should be initiated rapidly and can still result in a favorable neurologic outcome. Therefore, the course of this patient's care is a useful adjunct to the current literature for management of similar cases in hopes of a favorable outcome.

\section{Acknowledgements}

North Broward Hospital District

\section{References}

1. Go AS, Mozaffarian D, Roger VL, Benjamin EJ, Berry JD, et al. (2014) Heart disease and stroke statistics--2014 update: a report from the American Heart Association. Circulation 129: e28-292.

2. Pusswald G, Fertl E, Faltl M, Auff E (2000) Neurological rehabilitation of severely disabled cardiac arrest survivors. Part II. Life situation of patients and families after treatment. Resuscitation 47: 241-8.

3. Geocadin RG, Koenig MA, Jia X, Stevens RD, Peberdy MA (2008) Management of brain injury after resuscitation from cardiac arrest. Neurol Clin 26: 487-506.

4. Nolan JP, Morley PT, Vanden Hoek TL, Hickey RW, Kloeck WG, et al. (2003) Therapeutic hypothermia after cardiac arrest: an advisory statement by the advanced life support task force of the International Liaison Committee on Resuscitation. Circulation 108: 118-21.

5. Bernard SA, Gray TW, Buist MD, Jones BM, Silvester W, et al. (2002) Treatment of comatose survivors of out-of-hospital cardiac arrest with induced hypothermia. N Engl J Med 346: 557-63.

6. Hypothermia after Cardiac Arrest Study Group (2002) Mild therapeutic hypothermia to improve the neurologic outcome after cardiac arrest. N Engl J Med 346: 549-56.

7. Mooney MR, Unger BT, Boland LL, Burke MN, Kebed KY, et al. (2011) Therapeutic hypothermia after out-of-hospital cardiac arrest: evaluation of a regional system to increase access to cooling. Circulation 124: 206-14.

8. Choi DW (1992) Excitotoxic cell death. J Neurobiol 23: 1261-76.

9. Traystman RJ, Kirsch JR, Koehler RC (1991) Oxygen radical mechanisms of brain injury following ischemia and reperfusion. J Appl Physiol (1985) 71: 1185-95. 10. Vaagenes P, Ginsberg M, Ebmeyer U, Ernster L, Fischer M, et al. (1996) Cerebral resuscitation from cardiac arrest: pathophysiologic mechanisms. Crit Care Med 24(2 Suppl): S57-68.

11. Chan PH (1996) Role of oxidants in ischemic brain damage. Stroke 27: 1124-9. 
12. Chan PH (2001) Reactive oxygen radicals in signaling and damage in the ischemic brain. J Cereb Blood Flow Metab 21: 2-14.

13. Herlitz J, Svensson L, Engdahl J, Angquist KA, Silfverstolpe J, et al. (2006) Association between interval between call for ambulance and return of spontaneous circulation and survival in out-of-hospital cardiac arrest. Resuscitation 71: 40-6.

14. Nielsen N, Hovdenes J, Nilsson F, Rubertsson S, Stammet P, et al. (2009) Outcome, timing and adverse events in therapeutic hypothermia after out-of-hospital cardiac arrest. Acta Anaesthesiol Scand 53: 926-34.

15. Oddo M, Schaller MD, Feihl F, Ribordy V, Liaudet L (2006) From evidence to clinical practice: effective implementation of therapeutic hypothermia to improve patient outcome after cardiac arrest. Crit Care Med 34: 1865-73.

16. Sasson C, Rogers MA, Dahl J, Kellermann AL (2010) Predictors of survival from out-of-hospital cardiac arrest: a systematic review and meta-analysis. Circ Cardiovasc Qual Outcomes 3: 63-81.

17. Arrich J, Holzer M, Havel C, Müllner M, Herkner H (2012) Hypothermia for neuroprotection in adults after cardiopulmonary resuscitation. Cochrane Database Syst Rev 9: CD004128.

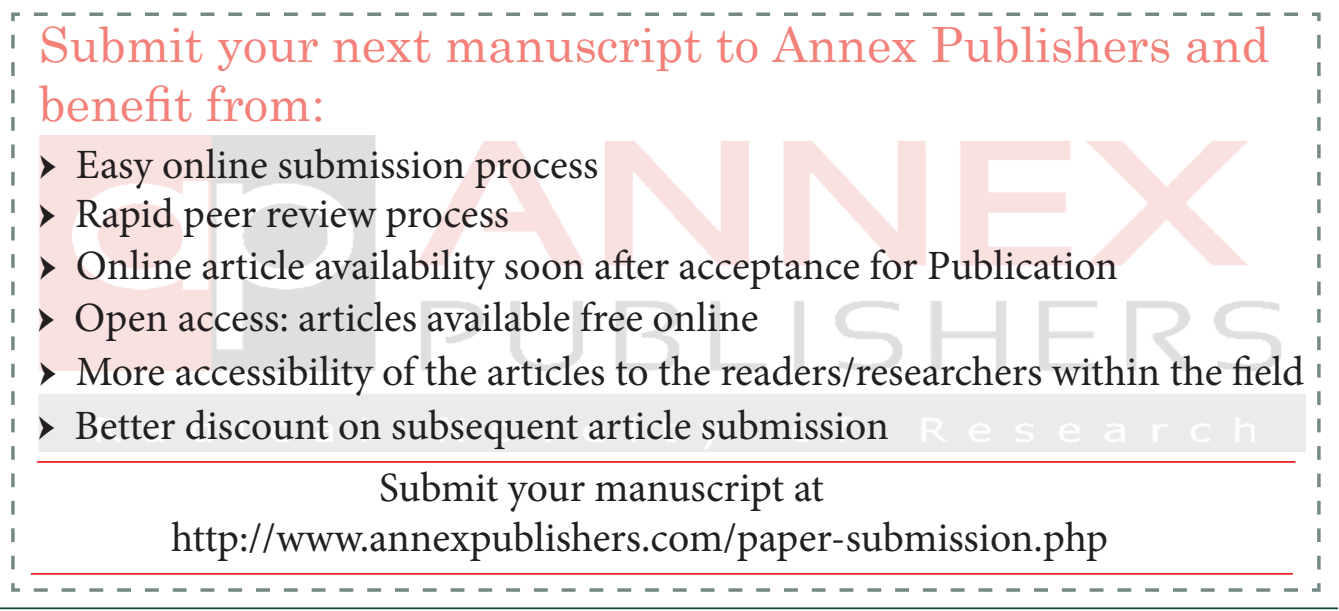

